\title{
Schauder Bases for Symmetric Tensor Products
}

\author{
By \\ Bogdan C. GReCU* and Raymond A. RyAN*
}

\begin{abstract}
For a Banach space $E$ with Schauder basis, we prove that the $n$ fold symmetric tensor product $\hat{\otimes}_{\mu, s}^{n} E$ has a Schauder basis for all symmetric uniform crossnorms $\mu$. This is done by modifying the square ordering on $\mathbb{N}^{n}$ and showing that the new ordering gives tensor product bases in both $\hat{\otimes}_{\mu}^{n} E$ and $\hat{\otimes}_{\mu, s}^{n} E$.

The main purpose of this article is to prove that the $n$-fold symmetric tensor product of a (real or complex) Banach space $E$ has a Schauder basis whenever $E$ does. The result was stated without proof in Ryan's thesis [11] and has been constantly referred to in the literature. In the particular case of a shrinking Schauder basis for a complex Banach space $E$, an implicit proof was given by Dimant and Dineen [2]. The existence of a basis for the full tensor product was proved by Gelbaum and Gil de Lamadrid [7] who also showed that the unconditionality of the basis for $E$ does not necessarily imply the same property for the tensor product basis. This was taken further by Kwapien and Pełczyński [8] who treated this issue in the context of spaces of matrices and by Pisier [9] and Schütt [12]. The dual problem, whether the monomials are a basis in the space of homogeneous polynomials, was dealt with by Dimant in her thesis [1], as well as in two other articles, together with Dineen [2] and Zalduendo [3]. The unconditionality (or lack thereof) of the monomial basis was extensively analysed by Defant, Díaz, Garcia and Maestre [4].
\end{abstract}

Communicated by H. Okamoto. Received November 25, 2003. Revised June 3, 2004.

2000 Mathematics Subject Classification(s): Primary 46B28, Secondary 46B15.

Key words and phrases: Schauder basis, tensor product.

* Department of Mathematics, National University of Ireland, Galway, Ireland.

e-mail: bogdan@wuzwuz.nuigalway.ie

e-mail: ray.ryan@nuigalway.ie

(c) 2005 Research Institute for Mathematical Sciences, Kyoto University. All rights reserved. 


\section{$\S 1$. Preliminaries}

Let $E$ and $F$ be (real or complex) Banach spaces and let $E \otimes F$ be their tensor product. A norm $\mu$ on $E \otimes F$ is said to be a reasonable crossnorm on $E \otimes F$ if

1. $\mu(x \otimes y) \leq\|x\|\|y\|$ for every $x \in E$ and $y \in F$

2. for every $\varphi \in E^{*}$ and $\psi \in F^{*}$, the linear functional $\varphi \otimes \psi$ on $E \otimes_{\mu} F$ is bounded and $\|\varphi \otimes \psi\| \leq\|\varphi\|\|\psi\|$.

The projective and the injective norms $\pi$ and $\varepsilon$ satisfy these conditions and it can be shown that a norm $\mu$ on $E \otimes F$ is a reasonable crossnorm if and only if $\varepsilon(u) \leq \mu(u) \leq \pi(u)$ for every $u \in E \otimes F$ (see [5], [10] for details).

A uniform crossnorm is an assignment to each pair $E$ and $F$ of Banach spaces of a reasonable crossnorm on $E \otimes F$ which behaves well with respect to the formation of tensor product of operators, in the sense that if $S: E \rightarrow X$ and $T: F \rightarrow Y$ are bounded linear operators then $S \otimes T: E \otimes F \rightarrow X \otimes Y$, defined by $S \otimes T(x \otimes y)=S x \otimes T y$, is bounded and $\|S \otimes T\| \leq\|S\|\|T\|$. In what follows we work with a uniform crossnorm $\mu$ and with $E \hat{\otimes}_{\mu} F$, the completion of $E \otimes F$ in this norm.

Let $\mathbb{N}$ be the set of nonnegative integers. A sequence $\left\{e_{i}\right\}_{i \in \mathbb{N}}$ is a Schauder basis for the normed space $E$ if every element $x$ of $E$ can be uniquely represented as $x=\sum_{i \in \mathbb{N}} x_{i} e_{i}$. If the convergence of the series is unconditional for all $x$ then the basis is called unconditional. It is well known that $\left\{e_{i}\right\}_{i \in \mathbb{N}}$ is a Schauder basis if and only if $\overline{\operatorname{span}}\left\{e_{i}\right\}=E$ and $\left\{e_{i}\right\}_{i \in \mathbb{N}}$ is a basic sequence: there exists a constant $C$ such that $\left\|\sum_{i=1}^{n} a_{i} e_{i}\right\| \leq C\left\|\sum_{i=1}^{n+p} a_{i} e_{i}\right\|$ for all natural numbers $n, p$ and every set of scalars $\left\{a_{i}\right\}_{i \in \mathbb{N}}$. This amounts to saying that the projections $P_{n}\left(\sum_{i \in \mathbb{N}} x_{i} e_{i}\right)=\sum_{i=1}^{n} x_{i} e_{i}$ of $E$ on the span of the first $n$ basis vectors satisfy $\left\|P_{n}\right\| \leq C$ for all $n$. The smallest of such constants $C$ is called the basis constant of $\left\{e_{i}\right\}_{i \in \mathbb{N}}$. The linear functionals $e_{k}^{*}\left(\sum_{i \in \mathbb{N}} x_{i} e_{i}\right)=x_{k}$ are bounded and $\left\{e_{k}^{*}\right\}_{k \in \mathbb{N}}$ is a basic sequence in $E^{*}$.

If $\left\{e_{i}\right\}_{i \in \mathbb{N}}$ and $\left\{f_{j}\right\}_{j \in \mathbb{N}}$ are Schauder bases for $E$ and $F$ respectively, a natural question is: does the space $E \hat{\otimes}_{\mu} F$ have a Schauder basis? Since $\mu$ is a reasonable crossnorm, it is clear that $\overline{\operatorname{span}}\left\{e_{i} \otimes f_{j}\right\}_{(i, j) \in \mathbb{N} \times \mathbb{N}}=E \hat{\otimes}_{\mu} F$. The only thing needed then is an order on $\mathbb{N} \times \mathbb{N}$ with respect to which $\left\{e_{i} \otimes f_{j}\right\}_{(i, j) \in \mathbb{N} \times \mathbb{N}}$ is a basic sequence. 
If we arrange $\left\{e_{i} \otimes f_{j}\right\}_{(i, j) \in \mathbb{N} \times \mathbb{N}}$ in a matrix, one classical way of ordering them is the so called diagonal ordering:

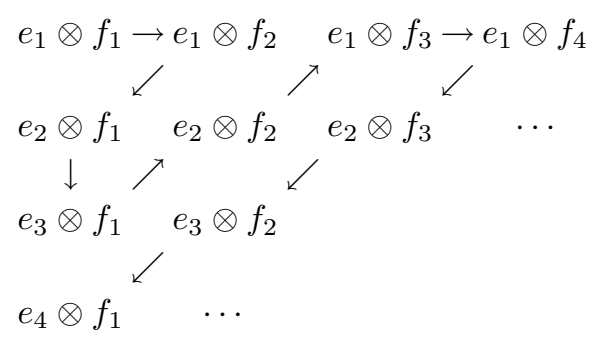

Among the set of all projections we encounter the main triangle projections

$$
T_{n}(u)=\sum_{i+j \leq n+1} a_{i j} e_{i} \otimes f_{j}
$$

for $u=\sum_{i, j} a_{i j} e_{i} \otimes f_{j}$, a finite linear combination of tensors of the form $e_{i} \otimes$ $f_{j}$. Kwapień and Pełczyński [8] proved that for $E=\ell_{p}$ and $F=\ell_{q}$ with $1 / p+1 / q \geq 1$ and the injective norm $\varepsilon$, the projections $T_{n}$ are unbounded, since $\left\|T_{n}\right\| \geq C(p, q) \ln n$. Therefore the diagonal ordering will not give a basic sequence.

Nevertheless, it is possible to order $\left\{e_{i} \otimes f_{j}\right\}_{(i, j) \in \mathbb{N} \times \mathbb{N}}$ in a satisfactory way. Gelbaum and Gil de Lamadrid [7] introduced the square ordering illustrated in the diagram below

$$
\begin{array}{rcc}
e_{1} \otimes f_{1} \rightarrow e_{1} \otimes f_{2} & e_{1} \otimes f_{3} & e_{1} \otimes f_{4} \\
\downarrow & \downarrow & \downarrow \\
e_{2} \otimes f_{1} \leftarrow e_{2} \otimes f_{2} & e_{2} \otimes f_{3} & e_{2} \otimes f_{4} \\
& \downarrow & \downarrow \\
e_{3} \otimes f_{1} \leftarrow e_{3} \otimes f_{2} \leftarrow e_{3} \otimes f_{3} & e_{3} \otimes f_{4} \\
& & \downarrow \\
\cdots & \leftarrow e_{4} \otimes f_{3} \leftarrow e_{4} \otimes f_{4}
\end{array}
$$

Their proofs also work with the following slightly modified ordering in which the role of rows and columns is reversed: $e_{1} \otimes f_{1}, e_{2} \otimes f_{1}, e_{2} \otimes f_{2}, e_{1} \otimes f_{2}$, $e_{3} \otimes f_{1}, \ldots$, and to which we will refer to as the square ordering. They proved that this ordering gives rise to a basic sequence. Let $P_{(i, j)}$ be the projection on the linear span of the first $(i, j)$ basis vectors with respect to the square ordering on $\mathbb{N} \times \mathbb{N}$. These projections are uniformly bounded; if $C$ and $D$ are the basis constants for $E$ and $F$, then the tensor product basis has a basis constant at most $5 C D$. 
The square ordering can be extended recursively to tensor products of any number of spaces, since the tensor product is associative and so $E \otimes F \otimes G$ may be identified with $(E \otimes F) \otimes G$.

When $E=F$ we can consider the symmetric tensor product $E \hat{\otimes}_{\mu, s} E$ as a subspace of the full tensor product $E \hat{\otimes}_{\mu} E$. In general the $n$-fold symmetric tensor product $\hat{\otimes}_{s}^{n} E$ is defined as $S\left(\hat{\otimes}^{n} E\right)$, where

$$
S\left(x_{1} \otimes \cdots \otimes x_{n}\right)=\frac{1}{n !} \sum_{\sigma \in S_{n}} x_{\sigma(1)} \otimes \cdots \otimes x_{\sigma(n)},
$$

$S_{n}$ being the group of permutations of the set $\{1, \ldots, n\}$. It is reasonable to expect that the corresponding square ordering

$$
\begin{aligned}
& e_{1} \otimes e_{1} \\
& e_{2} \otimes_{s} e_{1} \rightarrow e_{2} \otimes e_{2} \\
& e_{3} \otimes_{s} e_{1} \rightarrow e_{3} \otimes_{s} e_{2} \rightarrow e_{3} \otimes e_{3} \\
& e_{4} \otimes_{s} e_{1} \rightarrow e_{4} \otimes_{s} e_{2} \rightarrow e_{4} \otimes_{s} e_{3} \rightarrow \cdots
\end{aligned}
$$

will make $\left\{e_{i} \otimes_{s} e_{j}\right\}_{j \leq i}$ a Schauder basis for $E \hat{\otimes}_{\mu, s} E$. Ryan stated this in his thesis [11] but it was not actually proved. Given the fact that the symmetric tensor product is not defined recursively, one difficulty one might expect to encounter is how to describe this ordering in the case of $\hat{\otimes}_{\mu, s}^{n} E$. Therefore it seems more natural to obtain it from the ordering we already have in the full tensor product $\hat{\otimes}_{\mu}^{n} E$, in which case one might expect that the corresponding projections in the two spaces will be related. Let us see what happens for $n=2$. For a finite linear combination $u=\sum_{j \leq i} a_{i j} e_{i} \otimes_{s} e_{j}$ put

$$
\Pi_{(m, n)}(u)=\sum_{\substack{(i, j) \leq(m, n) \\ j \leq i}} a_{i j} e_{i} \otimes_{s} e_{j}
$$

We have, for example

$$
\Pi_{(3,1)}(u)=\sum_{\substack{(i, j) \leq(3,1) \\ j \leq i}} a_{i j} e_{i} \otimes_{s} e_{j}=P_{(3,1)} u+\frac{1}{2} a_{31} e_{1} \otimes e_{3} .
$$

The greater the index $(m, n)$ will be, the more terms the difference between the two projections will contain, so the more difficult will be to show that $\Pi_{(m, n)}$ are uniformly bounded. Nevertheless, it is hard to imagine that the ordering described above will not give a basic sequence. In fact this has been proved, for the projective norm, via the dual space of $\hat{\otimes}_{\pi, s}^{n} E$, for a complex Banach space 
$E$ by Dimant and Dineen [2]. Our goal is to give a proof that works for both real and complex spaces and uses only tensors.

\section{§2. A Different Ordering}

In the sequel, for every positive integer $n$, we will call an element of $\mathbb{N}^{n}$ an $n$-multi-index.

The reason why there was a difference between $\Pi_{(3,1)}$ and $P_{(3,1)}$ is that in the square ordering, between $e_{1} \otimes e_{3}$ and $e_{3} \otimes e_{1}$ we come across other tensors: $e_{3} \otimes e_{2}, e_{3} \otimes e_{3}$ and $e_{2} \otimes e_{3}$. We circumvent this by introducing a new ordering, under which the permutations of the same multi-index stay together.

If $\alpha$ is an $n$-multi-index, we denote by $\alpha_{d}$ the multi-index obtained by arranging the elements of $\alpha$ in decreasing order. If $\alpha$ and $\beta$ are two decreasing multi-indices, we say that $\alpha<\beta$ if $\alpha_{1}=\max \alpha<\beta_{1}=\max \beta$ or $\alpha_{1}=\beta_{1}$ and $\left(\alpha_{2}, \alpha_{3}, \ldots, \alpha_{n}\right)<\left(\beta_{2}, \beta_{3}, \ldots, \beta_{n}\right)$.

Now if $\alpha$ and $\beta$ are arbitrary we say that $\alpha<\beta$ if

1. $\alpha_{d}<\beta_{d}$ or

2. $\alpha_{d}=\beta_{d}$, case in which $\alpha$ is a permutation of $\beta$, and the greater of the two will be the index for which $\max \alpha=\max \beta$ appears earlier. If $\max \alpha$ appears in the same position for both of the multi-indices, we eliminate it and compare the remaining $(n-1)$-multi-indices.

When $n=3$ the order is

$$
\begin{aligned}
&(1,1,1)<(1,1,2)<(1,2,1)<(2,1,1)<(1,2,2)<(2,1,2)<(2,2,1) \\
&<(2,2,2)<(1,1,3)<(1,3,1)<(3,1,1)<(1,2,3)<(2,1,3)<(1,3,2) \\
&<(2,3,1)<(3,1,2)<(3,2,1)<(2,2,3)<(2,3,2)<(3,2,2)<(1,3,3) \ldots
\end{aligned}
$$

It is easy to check that this is a total order.

\section{$\S 3 . \quad$ Main Results}

In what follows we will write $e_{\alpha}=e_{\alpha_{1}} \otimes e_{\alpha_{2}} \otimes \cdots \otimes e_{\alpha_{n}}$ for every $n$ multi-index $\alpha$. Let $P_{\alpha}^{n}: \hat{\otimes}_{\mu}^{n} E \rightarrow \hat{\otimes}_{\mu}^{n} E$ be the projection of $\hat{\otimes}_{\mu}^{n} E$ on the linear span of the first $\alpha$ basis vectors with respect to the ordering of $\mathbb{N}^{n}$ defined above. For a positive integer $k$, the operator $e_{k}^{*} \otimes e_{k}: E \rightarrow E$ is defined by $e_{k}^{*} \otimes e_{k}\left(\sum_{i \in \mathbb{N}} x_{i} e_{i}\right)=x_{k} e_{k}$. 
For a permutation $\sigma$ in $S_{n}$ let us put $\left(x_{1} \otimes \cdots \otimes x_{n}\right)^{\sigma}=x_{\sigma(1)} \otimes \cdots \otimes x_{\sigma(n)}$ and extend it by linearity and continuity to the whole of $\hat{\otimes}_{\mu}^{n} E$. There exist norms, called symmetric [5], such as $\pi$ and $\varepsilon$, for which $\mu\left(u^{\sigma}\right)=\mu(u)$ for all tensors $u$ and all $\sigma$ in $S_{n}$. Nevertheless, this does not happen in general, for instance the Chevet-Saphar norms $d_{p}$ and $g_{p}$ do not have this property (see [10] for details).

Proposition. Let $E$ be a Banach space with Schauder basis $\left\{e_{i}\right\}_{i \in \mathbb{N}}$ and $\mu$ a symmetric uniform crossnorm. Then the sequence $\left\{e_{\alpha}\right\}_{\alpha \in \mathbb{N}^{n}}$ with the ordering defined above is a Schauder basis for $\hat{\otimes}_{\mu}^{n} E$.

Proof. Obviously $\overline{\operatorname{span}}\left\{e_{\alpha}\right\}_{\alpha \in \mathbb{N}^{n}}=\hat{\otimes}_{\mu}^{n} E$. Therefore we need to show that $\left\{e_{\alpha}\right\}_{\alpha \in \mathbb{N}^{n}}$ is a basic sequence. We will prove it by induction. Let $C_{1}$ be the basis constant of $\left\{e_{i}\right\}_{i \in \mathbb{N}}$.

Let $n=2$. If $\alpha=(k, k)$ then $P_{\alpha}^{2}=P_{k}^{1} \otimes P_{k}^{1}$ and so $\left\|P_{\alpha}^{2}\right\| \leq C_{1}^{2}$. Now let $(k, k)<\alpha<(k+1, k+1)$. If $\alpha=(l, k+1)$ then

$$
P_{\alpha}^{2}=P_{(k, k)}^{2}+P_{l}^{1} \otimes\left(e_{k+1}^{*} \otimes e_{k+1}\right)+\left(e_{k+1}^{*} \otimes e_{k+1}\right) \otimes P_{l-1}^{1}
$$

and if $\alpha=(k+1, l)$ then

$$
P_{\alpha}^{2}=P_{(k, k)}^{2}+P_{l}^{1} \otimes\left(e_{k+1}^{*} \otimes e_{k+1}\right)+\left(e_{k+1}^{*} \otimes e_{k+1}\right) \otimes P_{l}^{1} .
$$

In both cases we obtain

$$
\left\|P_{\alpha}^{2}\right\| \leq C_{1}^{2}+2 C_{1}^{2}+2 C_{1}^{2}=5 C_{1}^{2},
$$

therefore the projections are uniformly bounded and so the basis constant for $\hat{\otimes}_{\mu}^{2} E$ is $C_{2} \leq 5 C_{1}^{2}$.

We suppose now that the result is true for all natural numbers less than $n-1$ and we prove it for $n$. As above, if $\alpha=(k, k, \ldots k)$ then $P_{\alpha}^{n}=\otimes^{n} P_{k}^{1}$ and so $\left\|P_{\alpha}^{n}\right\| \leq C_{1}^{n}$. Now let $(k, k, \ldots, k)<\alpha<(k+1, k+1, \ldots, k+1)$. We shall find an uniform bound for $\left\|P_{\alpha}^{n}\right\|$ moving along step by step, depending on the number of $(k+1)$ 's that appear in $\alpha$.

Suppose that $\alpha$ contains only one $k+1$ and that it appears in the last position. Then $\alpha=\left(\alpha_{1}, \ldots, \alpha_{n-1}, k+1\right)$ and

$$
\begin{aligned}
& P_{\alpha}^{n}=P_{(k, k, \ldots, k)}^{n} \\
& +\sum_{(k, k, \ldots, k)<\beta \leq\left(\alpha_{1}, \ldots \alpha_{n-1}, k+1\right)}\left(e_{\beta_{1}}^{*} \otimes e_{\beta_{1}}\right) \otimes \cdots \otimes\left(e_{\beta_{n-1}}^{*} \otimes e_{\beta_{n-1}}\right) \otimes\left(e_{\beta_{n}}^{*} \otimes e_{\beta_{n}}\right) .
\end{aligned}
$$

Now, unless $\alpha_{1}=\alpha_{2}=\ldots=\alpha_{n-1}=1$, there are multi-indices that contain $k+1$ on one of the $(n-1)^{t h}, \ldots, 1^{s t}$ positions and are less than $\alpha$. Consider all 
the multi-indices $\beta$ that are less than $\alpha$ and contain $k+1$ on the $n^{\text {th }}$ position and sum the corresponding terms in the expression above. We obtain $P_{\left(\alpha_{1}, \ldots \alpha_{n-1}\right)}^{n-1} \otimes$ $\left(e_{k+1}^{*} \otimes e_{k+1}\right)$. Now consider all the multi-indices that are less than $\alpha$ and contain $k+1$ on the $(n-1)^{t h}$ position. Because the ordering we are using is total, there exists a $\gamma$ in $\mathbb{N}^{n-1}$ such that $\left(\gamma_{1}, \ldots, \gamma_{n-2}, k+1, \gamma_{n-1}\right)$ is their maximum. Summing the corresponding terms in the expression of $P_{\alpha}^{n}$ we obtain

$$
\begin{aligned}
& \left(\sum\left(e_{\beta_{1}}^{*} \otimes e_{\beta_{1}}\right) \otimes \cdots \otimes\left(e_{k+1}^{*} \otimes e_{k+1}\right) \otimes\left(e_{\beta_{n}}^{*} \otimes e_{\beta_{n}}\right)\right)(u) \\
& =\left(\left(\sum_{\delta \leq \gamma}\left(e_{\delta_{1}}^{*} \otimes e_{\delta_{1}}\right) \otimes \cdots \otimes\left(e_{\delta_{n-1}}^{*} \otimes e_{\delta_{n-1}}\right) \otimes\left(e_{k+1}^{*} \otimes e_{k+1}\right)\right)\left(u^{\sigma}\right)\right)^{\sigma^{-1}} \\
& =\left(P_{\gamma}^{n-1} \otimes\left(e_{k+1}^{*} \otimes e_{k+1}\right)\left(u^{\sigma}\right)\right)^{\sigma^{-1}}
\end{aligned}
$$

where $\sigma$ is the transposition that takes $n$ to $n-1$. Since $\mu\left(u^{\sigma}\right)=\mu(u)$ for all $u$ in $\hat{\otimes}_{\mu}^{n} E$, we have

$$
\begin{aligned}
& \left\|\sum\left(e_{\beta_{1}}^{*} \otimes e_{\beta_{1}}\right) \otimes \cdots \otimes\left(e_{k+1}^{*} \otimes e_{k+1}\right) \otimes\left(e_{\beta_{n}}^{*} \otimes e_{\beta_{n}}\right)\right\| \\
& \quad=\left\|P_{\gamma}^{n-1} \otimes\left(e_{k+1}^{*} \otimes e_{k+1}\right)\right\| \leq C_{n-1}\left(2 C_{1}\right)
\end{aligned}
$$

with $C_{n-1}$ the basis constant for $\hat{\otimes}^{n-1} E$. Repeating the same procedure $n-2$ more times we obtain

$$
\left\|P_{\alpha}^{n}\right\| \leq C_{1}^{n}+n C_{n-1}\left(2 C_{1}\right)
$$

It is clear now that the same argument can be applied to all multi-indices that contain only one $k+1$ in any position, not necessarily the last.

The same technique can be used for all $\alpha$ that contain $l$ entries equal to $k+1$, where $1 \leq l \leq n-1$. There are $\left(\begin{array}{l}n \\ l\end{array}\right)$ possibilities to place those $(k+1)$ 's among the $n$ entries of $\alpha$. If we fix one of these $\left(\begin{array}{l}n \\ l\end{array}\right)$ arrangements and we sum the corresponding terms, we obtain, for an $(n-l)$-multi-index $\gamma$, the operator $\left(P_{\gamma}^{n-l} \otimes\left(e_{k+1}^{*} \otimes e_{k+1}\right)^{l}\right)^{\sigma^{-1}}$ with $\sigma$ the permutation that takes the positions where the $(k+1)$ 's appear in $\alpha$ to $(n-l+1, \ldots, n)$. The norm of each of these operators is bounded by $C_{n-l}\left(2 C_{1}\right)^{l}$. To obtain an upper bound for $\left\|P_{\alpha}^{n}\right\|$ we have to keep in mind that all the $n$ multi-indices that contain strictly less than $l$ entries equal to $k+1$ are less than $\alpha$. Therefore

$$
\left\|P_{\alpha}^{n}\right\| \leq C_{1}^{n}+n C_{n-1}\left(2 C_{1}\right)+\cdots+\left(\begin{array}{c}
n \\
l-1
\end{array}\right) C_{n-l+1}\left(2 C_{1}\right)^{l-1}+\left(\begin{array}{c}
n \\
l
\end{array}\right) C_{n-l}\left(2 C_{1}\right)^{l} .
$$


Continuing in the same manner until $l=n-1$, we get

$$
\left\|P_{\alpha}^{n}\right\| \leq \sum_{l=0}^{n-1}\left(\begin{array}{l}
n \\
l
\end{array}\right) C_{n-l}\left(2 C_{1}\right)^{l}
$$

for all $(k, k, \ldots, k)<\alpha<(k+1, k+1, \ldots, k+1)$ and for all $k$, which shows that $\left\{P_{\alpha}^{n}\right\}_{\alpha \in \mathbb{N}^{n}}$ is uniformly bounded and thus the sequence $\left\{e_{\alpha}\right\}_{\alpha \in \mathbb{N}^{n}}$ with the ordering defined above is a basic sequence, therefore a Schauder basis for $\hat{\otimes}_{\mu}^{n} E$.

Remark 1. The proof also shows that $C_{n} \leq \sum_{l=0}^{n-1}\left(\begin{array}{l}n \\ l\end{array}\right) C_{n-l}\left(2 C_{1}\right)^{l}$.

Let us denote by $\mathbb{N}_{d}^{n}$ the set of decreasing $n$-multi-indices: $\left\{\alpha \in \mathbb{N}^{n}: \alpha_{1} \geq\right.$ $\left.\alpha_{2} \geq \ldots \alpha_{n}\right\}$. Note that the restriction to $\mathbb{N}_{d}^{n}$ of the ordering we are working with is the same as the square ordering for $\mathbb{N}_{d}^{n}$, as explained in [6]. For an $\alpha$ in $\mathbb{N}_{d}^{n}$, let $\Pi_{\alpha}^{n}: \hat{\otimes}_{\mu, s}^{n} E \rightarrow \hat{\otimes}_{\mu, s}^{n} E$ be the projection of $\hat{\otimes}_{\mu, s}^{n} E$ on the linear span of the first $\alpha$ basis vectors with respect to the ordering of $\mathbb{N}_{d}^{n}$ in question.

Theorem. Let $E$ be a Banach space with Schauder basis $\left\{e_{i}\right\}_{i \in \mathbb{N}}$ and $\mu$ a symmetric uniform crossnorm. Then the sequence $\left\{e_{\alpha_{1}} \otimes_{s} e_{\alpha_{2}} \otimes_{s} \cdots \otimes_{s} e_{\alpha_{n}}\right\}_{\alpha \in \mathbb{N}_{d}^{n}}$ with the square ordering is a Schauder basis for $\hat{\otimes}_{\mu, s}^{n} E$.

Proof. As in the previous proposition, it suffices to show that $\left\{e_{\alpha_{1}} \otimes_{s}\right.$ $\left.e_{\alpha_{2}} \otimes_{s} \cdots \otimes_{s} e_{\alpha_{n}}\right\}_{\alpha \in \mathbb{N}_{d}^{n}}$ is a basic sequence, that is there exists a positive constant $C$ such that $\mu\left(\Pi_{\alpha}^{n} u\right) \leq C \mu\left(\Pi_{\beta}^{n} u\right)$ for every $\alpha \leq \beta$ in $\mathbb{N}_{d}^{n}$ and $u$ in $\hat{\otimes}_{\mu, s}^{n} E$.

Fix two decreasing multindices $\alpha$ and $\beta$ with $\alpha \leq \beta$. It is enough to prove the above inequality for tensors $u$ that are finite linear combinations in $\hat{\otimes}_{\mu, s}^{n} E$ of terms corresponding to indices no greater than $\beta$, in which case $\Pi_{\beta}^{n} u=u$. We have

$$
\begin{aligned}
\Pi_{\alpha}^{n} u & =\sum_{\substack{\gamma \in \mathbb{N}_{d}^{n} \\
\gamma \leq \alpha}} u_{\gamma_{1} \ldots \gamma_{n}} e_{\gamma_{1}} \otimes_{s} \cdots \otimes_{s} e_{\gamma_{n}} \\
& =\sum_{\substack{\gamma \in \mathbb{N}_{d}^{n} \\
\gamma \leq \alpha}} \sum_{\sigma \in S_{n}} \frac{1}{n !} u_{\gamma_{1} \ldots \gamma_{n}} e_{\gamma_{\sigma(1)}} \otimes \cdots \otimes e_{\gamma_{\sigma(n)}} .
\end{aligned}
$$

For $\gamma \leq \alpha$ and $\sigma \in S_{n}$, we have, by the definition of our ordering on $\mathbb{N}^{n}$, $\left(\gamma_{\sigma(1)}, \gamma_{\sigma(2)}, \ldots, \gamma_{\sigma(n)}\right) \leq\left(\gamma_{1}, \gamma_{2}, \ldots, \gamma_{n}\right) \leq \alpha$. On the other hand, if $\delta$ is a multi-index in $\mathbb{N}^{n}$ and $\delta \leq \alpha$ then $\delta_{d} \leq \alpha_{d}=\alpha$. Let $\tau$ be the permutation that takes $\delta_{d}$ to $\delta$. Then $e_{\delta}=e_{\delta_{1}} \otimes \cdots \otimes e_{\delta_{n}}=e_{\left(\delta_{d}\right)_{\tau(1)}} \otimes \cdots \otimes e_{\left(\delta_{d}\right)_{\tau(n)}}$ and so all 
the terms less than $\alpha$ of which $u$ is a linear combination in $\otimes_{\mu}^{n} E$ appear in the above sum. Therefore

$$
\Pi_{\alpha}^{n} u=P_{\alpha}^{n} u
$$

and

$$
\mu\left(\Pi_{\alpha}^{n} u\right)=\mu\left(P_{\alpha}^{n} u\right) \leq C_{n} \mu(u)=C_{n} \mu\left(\Pi_{\beta}^{n} u\right)
$$

which shows that $\left\{e_{\alpha_{1}} \otimes_{s} e_{\alpha_{2}} \otimes_{s} \cdots \otimes_{s} e_{\alpha_{n}}\right\}_{\alpha \in \mathbb{N}_{d}^{n}}$ is a basic sequence with basis constant no greater that $C_{n}$, the basis constant for $\hat{\otimes}_{\mu}^{n} E$.

In the case when $\mu=\pi$, the projective norm, we have $\left(\hat{\otimes}_{\pi}^{n} E\right)^{*}=\mathcal{L}_{s}\left({ }^{n} E\right)$, the space of symmetric $n$-linear forms on $E$ with the usual sup norm. By the polarization formula [6], $\mathcal{L}_{s}\left({ }^{n} E\right)$ is isomorphic to $\mathcal{P}\left({ }^{n} E\right)$, the space of $n$-homogeneous polynomials on $E$ with the sup norm. For an $\alpha$ in $\mathbb{N}_{d}^{n}$, the functional $\left(e_{\alpha_{1}} \otimes_{s} e_{\alpha_{2}} \otimes_{s} \cdots \otimes_{s} e_{\alpha_{n}}\right)^{*}$ belongs to $\mathcal{L}_{s}\left({ }^{n} E\right)$ and

$$
\begin{aligned}
& \left(e_{\alpha_{1}} \otimes_{s} e_{\alpha_{2}} \otimes_{s} \cdots \otimes_{s} e_{\alpha_{n}}\right)^{*}\left(x_{1}, \ldots, x_{n}\right) \\
& \quad=\left(e_{\alpha_{1}} \otimes_{s} e_{\alpha_{2}} \otimes_{s} \cdots \otimes_{s} e_{\alpha_{n}}\right)^{*}\left(x_{1} \otimes_{s} \cdots \otimes_{s} x_{n}\right) \\
& \quad=n !\left(e_{\alpha_{1}}^{*} \otimes_{s} e_{\alpha_{2}}^{*} \otimes_{s} \cdots \otimes_{s} e_{\alpha_{n}}^{*}\right)\left(x_{1} \otimes_{s} \cdots \otimes_{s} x_{n}\right) .
\end{aligned}
$$

Thus the sequence $\left\{e_{\alpha_{1}}^{*} \otimes_{s} e_{\alpha_{2}}^{*} \otimes_{s} \cdots \otimes_{s} e_{\alpha_{n}}^{*}\right\}_{\alpha \in \mathbb{N}_{d}^{n}}$ is a basic sequence in $\mathcal{L}_{s}\left({ }^{n} E\right)$, and so is $\left\{x_{\alpha}\right\}_{\alpha \in \mathbb{N}_{d}^{n}}$, with $x_{\alpha}=x_{\alpha_{1}} x_{\alpha_{2}} \cdots x_{\alpha_{n}}$, in $\mathcal{P}\left({ }^{n} E\right)$. The polynomials $x_{\alpha}$ are called the monomials of degree $n$. Therefore the monomials will form a basis for their closed linear span. If $n=1$ then this closed linear span is the whole of $\mathcal{P}\left({ }^{1} E\right)=E^{*}$ if and only if the basis $\left\{e_{i}\right\}_{i \in \mathbb{N}}$ is shrinking, in which case the closed linear span of the monomials of degree $n$ is $\mathcal{P}_{w}\left({ }^{n} E\right)$, the space of $n$-homogeneous polynomials that are weakly continuous on bounded sets. Thus we have the following

Corollary. If the (real or complex) Banach space E has a shrinking Schauder basis then the monomials of degree $n$ with the square ordering of $\mathbb{N}_{d}^{n}$ form a Schauder basis for $\mathcal{P}_{w}\left({ }^{n} E\right)$.

For complex spaces $E$ this result has been proved, in a different way, by Dimant and Dineen [2]. We note than the corollary and the theorem (for the projective norm) are equivalent, due to the duality between the bases $\left\{e_{\alpha_{1}} \otimes_{s}\right.$ $\left.e_{\alpha_{2}} \otimes_{s} \cdots \otimes_{s} e_{\alpha_{n}}\right\}_{\alpha \in \mathbb{N}_{d}^{n}}$ and $\left\{n ! e_{\alpha_{1}}^{*} \otimes_{s} e_{\alpha_{2}}^{*} \otimes_{s} \cdots \otimes_{s} e_{\alpha_{n}}^{*}\right\}_{\alpha \in \mathbb{N}_{d}^{n}}$.

Remark 2. The antisymmetrization operator $A: \otimes^{n} E \rightarrow \otimes^{n} E$ is defined by

$$
A\left(x_{1} \otimes \cdots \otimes x_{n}\right)=\frac{1}{n !} \sum_{\sigma \in S_{n}} \operatorname{sgn}(\sigma) x_{\sigma(1)} \otimes \cdots \otimes x_{\sigma(n)} .
$$


The range of $A$ is called the space of alternating tensors $\otimes_{a}^{n} E=A\left(\otimes^{n} E\right)$. We write

$$
x_{1} \otimes_{a} \cdots \otimes_{a} x_{n}=A\left(x_{1} \otimes \cdots \otimes x_{n}\right) .
$$

Restricting the order defined in section 2 to the set $\mathbb{N}_{s d}^{n}=\left\{\alpha \in \mathbb{N}^{n}: \alpha_{1}>\alpha_{2}>\right.$ $\left.\ldots>\alpha_{n}\right\}$, and copying word for word the proof of the theorem, we obtain that the sequence $\left\{e_{\alpha_{1}} \otimes_{a} e_{\alpha_{2}} \otimes_{a} \cdots \otimes_{a} e_{\alpha_{n}}\right\}_{\alpha \in \mathbb{N}_{s d}^{n}}$ is a Schauder basis for $\hat{\otimes}_{\mu, a}^{n} E$.

Remark 3. In the case of the basis $\left\{e_{i}\right\}_{i \in \mathbb{N}}$ being unconditional, a natural question seems to be: will the bases $\left\{e_{\alpha}\right\}_{\alpha \in \mathbb{N}^{n}}$ or $\left\{e_{\alpha_{1}} \otimes_{s} e_{\alpha_{2}} \otimes_{s} \cdots \otimes_{s} e_{\alpha_{n}}\right\}_{\alpha \in \mathbb{N}_{d}^{n}}$ be unconditional? It turns out that this is a very strong request. If the basis $\left\{e_{\alpha}\right\}_{\alpha \in \mathbb{N}^{n}}$ were unconditional then the main triangle projections would be bounded, which, as we have seen [8], is not true even for "nice" spaces like certain $\ell_{p}$ 's. Kwapień and Pełczyński also showed that for $\ell_{2} \otimes \ell_{2}$, considered as a space of matrices whose entries are the coordinates of the elements of $\ell_{2} \otimes \ell_{2}$ relative to the basis $\left\{e_{(i, j)}\right\}_{(i, j) \in \mathbb{N}^{2}}$, endowed with a unitary matrix norm, the basis is unconditional if and only if the norm in question is equivalent to the Hilbertian norm on $\ell_{2} \otimes \ell_{2}$. Working for tensors product of two spaces $E \otimes F$ with a uniform crossnorm, Pisier [9] and Schütt [12] obtained independently that the tensor product basis is unconditional if and only if $E \hat{\otimes}_{\mu} F$ has the Gordon-Lewis property. Their results have been extended to $n$-fold symmetric and full tensor products by Defant, Díaz, Garcia and Maestre [4]. Considering the dual problem, that is the spaces $\mathcal{P}_{w}\left({ }^{n} E\right)$ and $\mathcal{P}\left({ }^{n} E\right)$ having unconditional basis, they narrowed down considerably the list of candidates $E$ with this property, showing that it does not happen for any Banach space that contains uniformly complemented one of the sequences $\left(\ell_{p}^{n}\right)_{n \in \mathbb{N}}$ with $1<p \leq \infty$. Dineen [6] conjectures that the answer is going to be affirmative "rarely and perhaps never".

\section{Acknowledgments}

Both authors wish to acknowledge the financial support of an Enterprise Ireland Basic Research Grant.

\section{References}

[1] Dimant, V., Bases de Schauder en Espacios de Polinomios, doctoral thesis, Universidad de San Andres, Buenos Aires, 1996.

[2] Dimant, V. and Dineen, S., Banach subspaces of spaces of holomorphic mappings and related topics, Math. Scand., 83 (1998), 142-160. 
[3] Dimant, V. and Zalduendo, I., Bases in Spaces of Multilinear Forms over Banach Spaces, J. Math. Anal. Appl., 200 (1996), 548-566.

[4] Defant, A., Díaz, J. C., Garcia, D. and Maestre, M., Unconditional Basis and GordonLewis Constants for Spaces of Polynomials, J. Funct. Anal., 181 (2001), 119-145.

[5] Defant, A. and Floret, K., "Tensor Norms and Operator Ideals", North-Holland, Amsterdam, London, New York, Tokyo, 1993.

[6] Dineen, S., "Complex Analysis on Infinite Dimensional Spaces", Springer Monogr. Math., Springer-Verlag, London, 1999.

[7] Gelbaum, B. R. and Gil de Lamadrid, J., Bases of tensor products of Banach spaces, Pacific J. Math., 11 (1961), 1281-1286.

[8] Kwapień, S. and Pełczyński, A., The main triangle projection in matrix spaces and its applications, Studia Math., 34 (1970), 43-68.

[9] Pisier, G., Some result on Banach spaces without unconditional structure, Compositio Math., 37 (1978), 3-19.

[10] Ryan, R. A., "Introduction to Tensor Products of Banach Spaces", Springer Monogr. Math., Springer-Verlag, London, 2001.

[11] _ Application of Topological Tensor Products to Infinite Dimensional Holomorphy, doctoral thesis, Trinity College Dublin, 1980.

[12] Schütt, C., Unconditionality in tensor products, Israel J. Math., 31 (1978), 209-216. 